Granulocyte-colony stimulating factor (G-CSF) increases the proliferation and maturation of committed polymorphonuclear leukocyte precursors, as well as the function of mature polymorphonuclear leukocytes. It has previously been shown in pediatric patients that G-CSF induces reconversion of fatty bone marrow to hematopoietic bone marrow in the pelvis and lower extremities that is detectable by magnetic resonance imaging (MRI). Here, we report a 13-year-old Burkitt leukemia patient with bone pain while he was in remission. He was on G-CSF after cessation of high-dose and low-dose cytarabine chemotherapy. He was suspected to have a leukemia relapse. Pelvic MRI was consistent with leukemic infiltration. However, the pathology of bone marrow biopsy resulted in normal findings. Thus it was suggested that concurrent administration of G-CSF could be the causative agent for both bone pain and false-positive MRI findings. The control MRI after interruption of G-CSF revealed normal findings. In conclusion, radiologists should be informed about the type of therapy, including G-CSF administration, in order to overcome misinterpretation of bone marrow MRI.

Key words: MRI, G-CSF, leukemia, child.

\section{False positivity of magnetic resonance imaging under the effect of granulocyte-colony stimulating factor in a child with leukemia}

\author{
Fatma Betul Cakir', Begumhan Baysal', Oner Dogan ${ }^{3}$
}

\author{
1Bezmialem Vakif University School of Medicine, Department of Pediatric Hematology- \\ Oncology, Istanbul, Turkey \\ ${ }^{2}$ Goztepe Educational and Research Hospital, Department of Radiodiagnostics, Istanbul, \\ Turkey \\ 3Istanbul University Istanbul Medical Faculty, Department of Pathology, Istanbul, Turkey
}

\section{Introduction}

Granulocyte-colony stimulating factor (G-CSF) increases the proliferation and maturation of committed polymorphonuclear leukocyte precursors, as well as the function of mature polymorphonuclear leukocytes. It is used as a primary prophylaxis immediately following a course of chemotherapy in a number of malignant tumors, including non-Hodgkin lymphomas [1]. It has previously been shown in pediatric patients with primary musculoskeletal tumors that G-CSF induces reconversion of fatty bone marrow to hematopoietic bone marrow in the pelvis and lower extremities that is detectable by magnetic resonance imaging (MRI) [1-6]. Awareness of the effect of G-CSF on bone marrow is important to avoid false-positive interpretations of marrow metastases. We report a Burkitt leukemia patient with false-positive pelvic MRI findings under concurrent G-CSF therapy.

\section{Case report}

A 13-year-old male patient was admitted to our pediatric hematologyoncology unit with pallor and malaise. His full blood count revealed WBC: $6300 / \mathrm{mm}^{3}$, Hgb: $9.2 \mathrm{~g} / \mathrm{dl}$, Plt: $70000 / \mathrm{mm}^{3}$ with no blasts in peripheral plate. The pathology and flow cytometry analysis of the bone marrow was consistent with Burkitt leukemia. The patient was stratified to be in group $C$ of the LMB 96 protocol. Granulocyte-colony stimulating factor was used as a primary prophylaxis after each chemotherapy course. He achieved remission after COPADM 1 (cyclophosphamide + vincristine + prednisolone + doxorubicin + + high-dose methotrexate + triple intrathecal therapy) chemotherapy. He had severe bone pain particularly at the pelvic girdle after CYVE-1 (high-dose and low-dose cytarabine) chemotherapy. His blood count was WBC: $24000 / \mathrm{mm}^{3}$ (72\% neutrophils, $28 \%$ bands), $\mathrm{Hgb}: 7.9 \mathrm{~g} / \mathrm{dl}$, Plt: $41000 / \mathrm{mm} 3$ ) at the $9^{\text {th }}$ day of G-CSF administration. Concurrently taken pelvic MRI suggested leukemia relapse. Coronal T1-weighted images obtained through both thighs showed diffuse low-signal change consistent with red marrow reconversion (Fig. 1). However, flow cytometry of bone marrow aspiration and the pathology of bone marrow biopsy taken from the MRI infiltration area just before CYVE-2 chemotherapy revealed no pathological results. Additionally, the control MRI of the pelvic area after interruption of G-CSF confirmed remission again (Fig. 2). Coronal T1-weighted images obtained through both thighs showed healthy marrow signal intensity. Granulocyte-colony stimulating factor was suggested as the causative agent both for bone pain and also for false positive MRI findings. He successfully finished his chemotherapy after 4 months without 


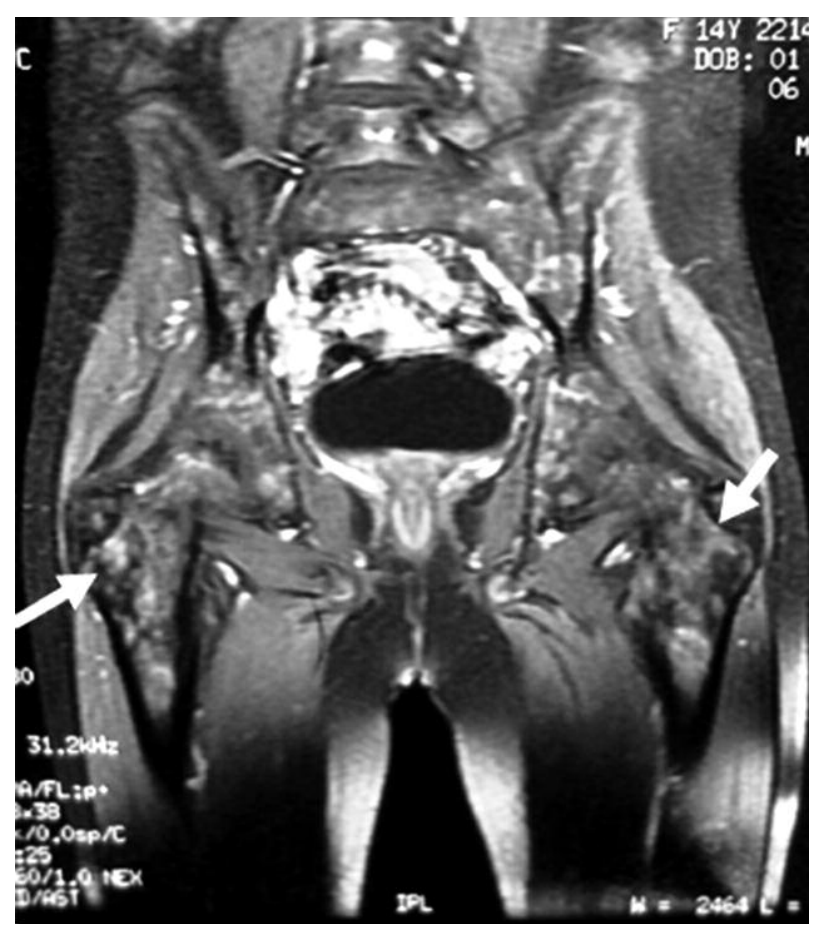

Fig. 1. T1-weighted images obtained through both thighs showing diffuse low-signal change consistent with red marrow reconversion

serious complications. Both pelvic MRI images and bilateral bone marrow biopsies verified complete remission. He has been in remission for 33 months.

\section{Discussion}

In this report, we described a Burkitt leukemia patient with MRI findings consistent with leukemia relapse due to G-CSF therapy. While hematopoietic marrow is found throughout the whole skeleton at birth, its conversion to fatty marrow occurs in limb bones extending from distal to proximal fashion over 20 years [7]. Neoplastic infiltration of the bone marrow results in reconversion of fatty to hematopoietic marrow in MRI images [2-7]. This reconversion is presumed to be attributable to the stimulation of residual hematopoietic cells within the predominantly fatty marrow. The reconversion process is also enhanced by administration of G-CSF [2-6]. Granulocyte-colony stimulating factor stimulates hematopoietic cells and increases their number and function to result in a change in marrow appearance that can be detected by MRI. Administering G-CSF to patients undergoing chemotherapy stimulates the marrow to produce more WBC. Since the MRI signal characteristics of highly cellular hematopoietic and neoplastic bone marrow are similar, differentiation between the reconverted normal hematopoietic marrow and recurrent tumor is not possible with conventional MRI techniques [2-6]. Magnetic resonance imaging of reconverted hematopoietic bone marrow revealed a decrease in the T1-weighted intensity under the effect of G-CSF [2-6]. Hence, alterations in pelvic MRI of our patient taken during G-CSF therapy simulated leukemic infiltration. Differentiating these changes without knowledge of the effect of G-CSF might be difficult. The pathological examination of bone marrow biopsy ruled out the possible cause of false pos-

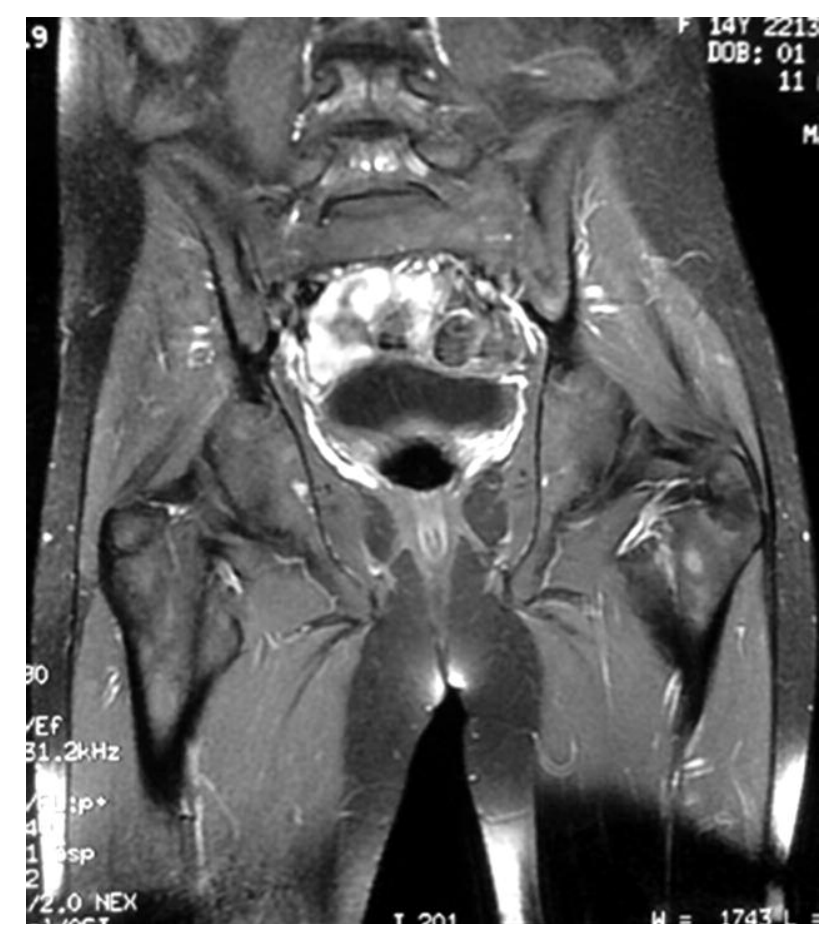

Fig. 2. T1-weighted images obtained through both thighs showing healthy marrow signal intensity

itivity in our patient. Red marrow reconversion in pediatric patients receiving G-CSF and the associated MRI findings have previously been reported in two studies describing 20 children with primary malignant musculoskeletal tumors [2, 3]. Fletcher et al. [2] reported focal and inhomogeneous alterations in bone marrow due to hematopoietic growth factorsupported chemotherapy. They observed changes consistent with reconversion of initially fatty marrow to red marrow in the pelvis and lower extremity in pediatric patients with a primary bone tumor. Seven of the 11 (64\%) patients had MRI changes consistent with reconversion to hematopoietic marrow with increases in absolute neutrophil counts. Ryan et al. [3] reported homogeneous but otherwise similar MRI findings in the lower extremities of children with osteosarcoma receiving G-CSF in addition to chemotherapy. Seven of 9 (77\%) patients with osteosarcoma who had received G-CSF in addition to chemotherapy had changes consistent with reconversion on MRI images. In an adult study, 10 (40\%) of the 25 patients with MRI scans available before and after G-CSF showed changes consistent with red marrow reconversion [5]. Areas of red marrow showed a decreased T1 signal on T1-weighted scans. The same study established the time interval after the last G-CSF dose to the follow-up MRI as 0-42 days (mean, 9 days). In our study, MRI was obtained during G-CSF administration.

In conclusion, our study shows that G-CSF can cause changes in bone marrow simulating leukemia relapse on MRI. Radiologists should be informed about the type of therapy, including G-CSF administration, in order to overcome misinterpretation of bone marrow MRI changes.

The authors declare no conflict of interest. 


\section{References}

1. Lieschke GJ, Burgess AW. Granulocyte colony-stimulating factor and granulocyte-macrophage colony-stimulating factor. Part 2. N Engl J Med 1992; 327: 99-106.

2. Fletcher BD, Wall JE, Hanna SL. Effect of hematopoietic growth factors on MR images of bone marrow in children undergoing chemotherapy. Radiology 1993; 189: 745-51.

3. Ryan SP, Weinberger E, White KS, Shaw DW, Patterson K, Nazar-Stewart $\mathrm{V}$, Miser J. MR imaging of bone marrow in children with osteosarcoma: effect of granulocyte colony-stimulating factor. AJR Am J Roentgenol 1995; 165: 915-20.

4. Ciray I, Lindman H, Aström GK, Wanders A, Bergh J, Ahlström HK. Effect of granulocyte colony-stimulating factor (G-CSF)-supported chemotherapy on MR imaging of normal red bone marrow in breast cancer patients with focal bone metastases. Acta Radiol 2003; 44: 472-84.

5. Hartman RP, Sundaram M, Okuno SH, Sim FH. Effect of granulocytestimulating factors on marrow of adult patients with musculoskeletal malignancies: incidence and MRI findings. AJR Am J Roentgenol 2004; 183: 645-53.

6. Daldrup-Link HE, Henning T, Link TM. MR imaging of therapy-induced changes of bone marrow. Eur Radiol 2007; 17: 743-61.

7. Moore SG, Dawson KL. Red and yellow marrow in the femur: agerelated changes in appearance at MR imaging. Radiology 1990; 175: 219-23.

\section{Address for correspondence}

\section{Fatma Betul Cakir}

Bezmialem Vakif University School of Medicine, Department of Pediatric Hematology-Oncology,

Istanbul, Turkey

tel. 05304644994

e-mail: fbetulcakir@gmail.com

Submitted: $\quad 10.03 .2012$

Accepted: $\quad 23.05 .2012$ 